\title{
A tool for the evaluation of human lower arm injury: approach, experimental validation and application to safe robotics \\ B. Povse $\dagger$, S. Haddadin $\ddagger^{*}$, R. Belder $\S$, D. Koritnik $\dagger$ and T. Bajd $\Phi$
}

$\dagger R \& D$ department for automation, robotics and electronic instrumentation, Dax Electronic systems Company,Vreskovo 68, Trbovlje, Slovenia.E-mail: borut.dax@siol.net

$\ddagger$ Institute of Automatic Control, Leibniz Universität Hannover, Hanover, Germany

$\S$ Robotics and Mechatronics Center, DLR, Oberpfaffenhofen, Germany

\Laboratory of Robotics, Faculty of Electrical Engineering, University of Ljubljana, Trzaska 25, Ljubljana, Slovenia

(Accepted February 16, 2015. First published online: April 22, 2015)

\begin{abstract}
SUMMARY
This paper treats the systematic injury analysis of lower arm robot-human impacts. For this purpose, a passive mechanical lower arm (PMLA) was developed that mimics the human impact response and is suitable for systematic impact testing and prediction of mild contusions and lacerations. A mathematical model of the passive human lower arm is adopted to the control of the PMLA. Its biofidelity is verified by a number of comparative impact experiments with the PMLA and a human volunteer. The respective dynamic impact responses show very good consistency and support the fact that the developed device may serve as a human substitute in safety analysis for the described conditions. The collision tests were performed with two different robots: the DLR Lightweight Robot III (LWR-III) and the EPSON PS3L industrial robot. The data acquired in the PMLA impact experiments were used to encapsulate the results in a robot independent safety curve, taking into account robot's reflected inertia, velocity and impact geometry. Safety curves define the velocity boundaries on robot motions based on the instantaneous manipulator dynamics and possible human injury due to unforeseen impacts.
\end{abstract}

KEYWORDS: Human biomechanics; Man-machine systems; Robot dynamics; Control of robotic systems; Mechatronic systems.

\section{Introduction and Contribution}

In the near future, manufacturing in large scale industry and small and medium enterprises (SMEs) will look significantly different from the current state-of-the-art. Certainly significant amounts of tasks are automated, and industrial robots are used heavily across many domains. However, especially in assembly, the majority of tasks are still performed manually- at present. This is mainly due to the required expert knowledge and/or sensitive manipulation skills of humans to perform complex tasks, such as skilful assembly. Nevertheless, presumably even a greater deal of work will be accomplished by robots soon, as novel manipulators capable of safe and sensitive manipulation and interaction capabilities have entered the market. ${ }^{1-3}$ On the market side, the expectation regarding these novel interactive robotic devices is to revolutionize manufacturing as we know it today and, in particular, let people and robots work closely together. Designing robots for interaction has become a challenging subdomain in pHRI (physical human-robot interaction), leading to novel devices that have one thing in common: active and/or passive compliance together with lightweight mechanical design as

* Corresponding author. E-mail: sami.haddadin@irt.uni-hannover.de 
central paradigms. One very successful early example is the fully torque-controlled DLR/KUKA Lightweight robot family. ${ }^{1}$ The robots are equipped with joint torque sensors in each joint and have redundant position measurement (on the motor and link side). ${ }^{4}$ Their underlying control paradigm is the so-called "soft-robotics" concept, aiming at equipping the robot with high performance interaction control capabilities, such as impedance control and collision detection with reflex reaction. ${ }^{5,6}$ Further examples of this novel class of robots are Barrett's WAM Arm, ${ }^{2}$ Rethink Robotics Baxter, ${ }^{3}$ or also more recently the class of variable stiffness/impedance robots. ${ }^{7,8}$

Of course, the target domains envisaged for such novel solutions raise the key question of how to ensure human safety during pHRI. ${ }^{9}$ As the physical contact between human and robot is the primary potential cause of injury in pHRI, its fundamentals have to be well understood and according countermeasures for its prevention are to be found. In this context, a significant body of work was conducted in the field of human-robot interaction safety. ${ }^{10-14}$ However, human-robot collisions and the resulting injuries have only recently been systematically investigated for various blunt impacts in refs. [15], [16]. Despite several insights being provided in these investigations, the thorough evaluation and understanding of soft-tissue injury in robotics remains an open issue. Recent results in refs. [17], [18] give very general insights and, for the first time, a systematic analysis of soft-tissue injury in robotics. The underlying experiments targeted understanding abdominal tissue injury, and the authors derived a methodology for embedding injury data into the robot motion controller, which they termed safe motion unit (SMU). This concept is based on a general injury data representation, i.e. so-called safety curves. These encode any type of injury or pain indicator in the generalized mass-velocity plane, leading to dynamic thresholds that must not be exceeded during a robot's motion. In particular, they can be used to calculate the maximum velocity; an arbitrary point on the robot structure is allowed to move due to its instantaneous dynamics (reflected inertia) and curvature properties.

In this paper, we analyze possible human lower arm injury during close cooperation of small industrial robot manipulators and human workers. Relevant scenarios could involve the preparation of various subassemblies of the end-product by the robot, which are then passed to the human worker for final assembly. Collisions are predominantly expected between the robot's end-effector and the unconstrained lower arm, wrist, or hand of the human worker. ${ }^{19}$ Typically non-life-threatening injuries, such as hematoma or suffusions, may occur and are of primary interest. Ref. [20] proposed the use of the impact energy density (IED) to determine the occurrence of hematoma due to humanrobot collisions. Soft-tissue injuries occur at an IED greater than $2.52 \mathrm{~J} / \mathrm{cm}^{2},{ }^{21}$ while hematoma or suffusions may occur below this value. ${ }^{1}$

We make following concrete contributions to lower arm injury analysis in this paper.

(1) Design, control, and verification of an active mechanical lower arm dummy that can reproduce the local human mechanical impact response.

(2) Analysis and re-evaluation of the IED as an indicator for hematoma or suffusion and investigation of tensile stress as a laceration indicator with human volunteer testing.

(3) Analysis of human pain during lower arm and hand collisions.

(4) Generation of safety-curves in the mass-velocity space for general robot-independent representation of hematoma and laceration criteria for subsequent use in the SMU paradigm.

In the first contribution, we focus on designing and validating a mechanical, actively controlled lower arm dummy. Our device can show similar impact response to its human counterpart and is designed to experimentally analyze the impact behavior of a robot. For this, it is important to select an appropriate bio-mechanical injury indicator that can predict the occurrence of the considered injury and can indeed be measured with the mechanical device. This is especially useful for subsequent systematic evaluation with impact parameter variation (in particular, impact velocity, curvature, and mass).

The second and third contributions provide basic information/data regarding the impact energy densities and tensile stresses caused by a robot-human impact at varying impact velocity together with the corresponding injuries and/or pain at several impact locations. ${ }^{2}$ Thus, we also contribute to

\footnotetext{
${ }^{1}$ Fractures of the lower arm bones are expected only in a worst-case scenario, since clamping of the worker's body is unlikely to occur in appropriately arranged robot workspaces. In order to focus our analysis, we omit this case in the present work and refer to a previous analysis in ref. [22].

${ }^{2}$ Note that the human impact experiments presented in this paper were performed with a single volunteer. Obviously, impact tests with a single subject are too few to conclude general human injury characteristics.
} 


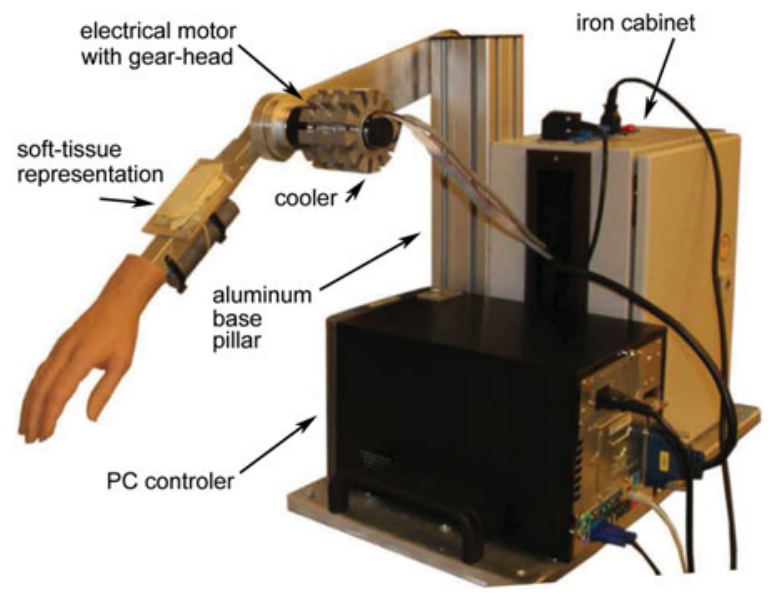

Fig. 1 The actively controlled passive mechanical lower arm PMLA.

the general agenda of collecting biomechanics safety data and provide it to the community, as well as currently ongoing standardization efforts.

The last contribution is the final step in human injury analysis in robotics. It aims at preparing any kind of impact data so that they can be used in robot motion control and planning.

\section{Approach and Experimental Setup}

\subsection{Passive (resting) mechanical lower arm}

To study the effect of an impact between a robot and the human lower arm in free space, an actively controlled PMLA was developed and equipped with inertial sensors (Fig. 1) for mimicking the dynamic response of the human lower arm. The first PMLA (version 1) had an inadequate impact response. ${ }^{23}$ The presented second version PMLA (hereinafter referred to as PMLA) incorporates a new prosthetic material for soft-tissue representation and a motorized elbow for elbow impedance emulation. The PMLA consists of a vertical base aluminium pillar to which the arm structure is attached, see Fig. 1.

The connection between the arm and the base consists of a passively adjustable shoulder joint. Two smaller aluminium profiles and a motor form the PMLA structure. The aluminium profile connected to the vertical aluminium base pillar represents the upper arm of length $280 \mathrm{~mm}$. The electrical motor with ceramic planetary gear-head connected to the aluminium upper arm represents the elbow joint. The motor is a Maxon RE 40 with integrated encoder (256-1024 C PT). A custom-made aluminium cooler is mounted on the motor in order to provide sufficient heat dissipation. The motor is driven by a Maxon servoamplifier (50 V/10 A). The aluminium lower arm structure supports a three-layer foam "sandwich" providing similar contact behavior to the relaxed muscle tissue. The length of the lower arm aluminium structure, measured from the mechanical elbow rotation center to the wrist, is 266 $\mathrm{mm}$. The total weight of the mechanical lower arm is $2.10 \mathrm{~kg}$. According to ref. [24], this value lies in the range of the mean lower arm weight value for women $(1.94 \mathrm{~kg})$ and men $(2.23 \mathrm{~kg})$. Finally, an iron cabinet with servoamplifier, power supply, and a PC is mounted onto the aluminium base plate.

2.1.1. Soft-tissue representation of the PMLA. In order to find suitable soft-tissue materials for mimicking the human mechanical response, a number of foam samples were experimentally compared via quasi-static testing to a human volunteer. For this, three different foam samples with various elastic properties were manufactured from prosthetic material (polyurethane foam). Figure 2 depicts the experimental setup for the volunteer test, which was the same for the foam samples. The lower arm of the volunteer was supported at the wrist and at the elbow. The robot end-effector was displaced incrementally into the soft tissue of the lower arm. The end-effector was positioned midway between

However, to our understanding they are necessary to show that in principle it is possible to replace human subject testing with adequately controlled mimicking devices based on as meaningful data as possible. 

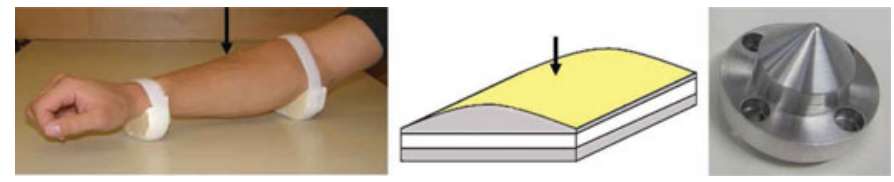

Fig. 2 From left to right: experimental setup for measurements of human soft tissue, three foam samples, and point end-effector.

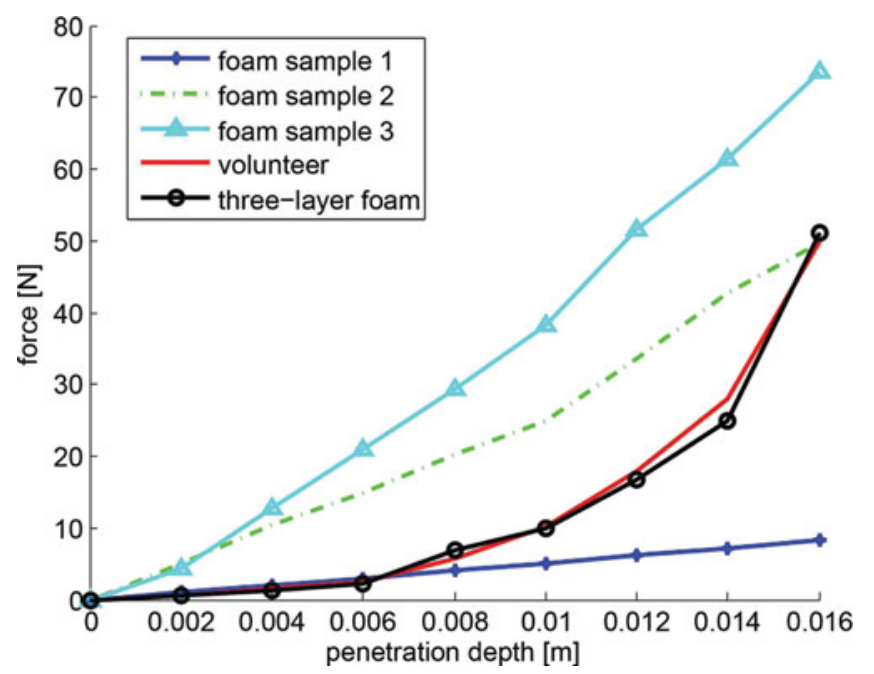

Fig. 3 Comparing the elastic deflection properties of human soft-tissue and three-layer foam "soft-tissue" representation.

the elbow and the wrist and displaced perpendicularly to the dorsal aspect of the lower arm surface. The quasi-static force between the robot end-effector and the lower arm was measured for each increment of the end-effector movement. In the experiment, a point end-effector (see Section 2.2) was used, since the point impact determines the most conservative criterion in terms of safety among the considered contact geometries.

The measurement results were used to construct a three-layer soft tissue representation using all three foam samples, determined with a trial-and-error procedure. Please note that later it is shown in Fig. 19 that this static fitting turned out to also generalize to the dynamic case, when we compare human and the PMLA for dynamic impacts with the LWR-III. However, we discuss the relevant data rather in the context of general human injury data in Section 3 than at this point with respect to the validation of the PMLA. The thickness of samples from the hardest to softest was 3, 7, and $4 \mathrm{~mm}$, respectively. Contact forces as a function of indentation depth for the three-layer "sandwich" and the human lower arm soft-tissue are depicted in Fig. 3.

2.1.2. Modeling and control of the human elbow and lower arm. The elbow control loop of the PMLA implements a well-known lumped mathematical model of the human lower arm, ${ }^{25-27}$ see Fig. 4. The bio-mechanical model is fixed in the shoulder joint and includes two antagonistic muscles $\left(B_{1}, B_{2}\right.$ - muscle dampings; $K_{1}, K_{2}$ - muscle elasticities; $J_{1}, J_{2}$ - muscle inertias; $T_{1}$ - biceps inner torque generator that counterbalances gravity), lower arm inertia $(J)$, elbow elasticity $\left(K_{p}\right)$, elbow damping $\left(B_{p}\right)$, elbow friction $\left(F_{t r}\right)$, and gravitational force $\left(F_{g}\right)$.

$\varphi, \varphi_{1}, \varphi_{2}$ denote the lower arm and the antagonistic muscles position, respectively. A mechanical representation of the model is depicted in Fig. 5. According to ref. [25], the parameters of antagonistic muscles may be equalized in a first approximation $\left(K_{1}=K_{2}, B_{1}=B_{2}, J_{1}=J_{2}\right)$, which introduces only 


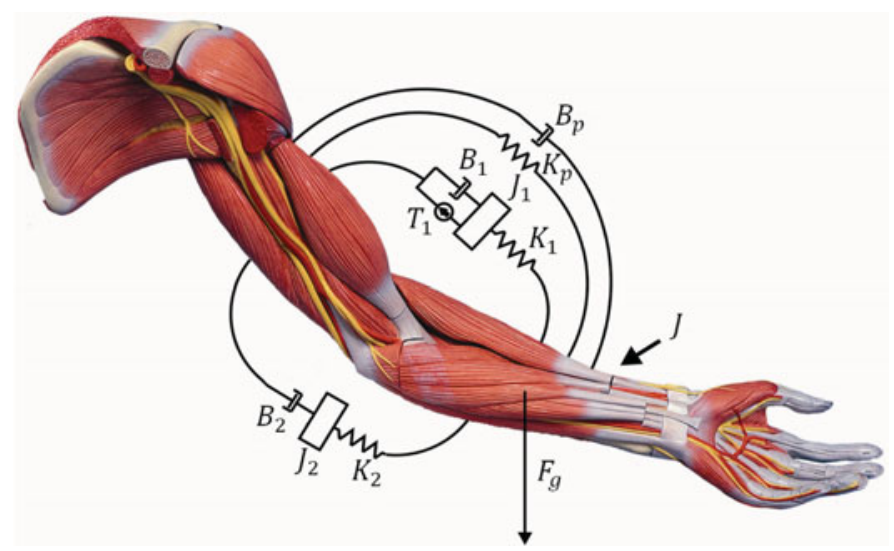

Fig. 4 A bio-mechanical model of the human lower arm.

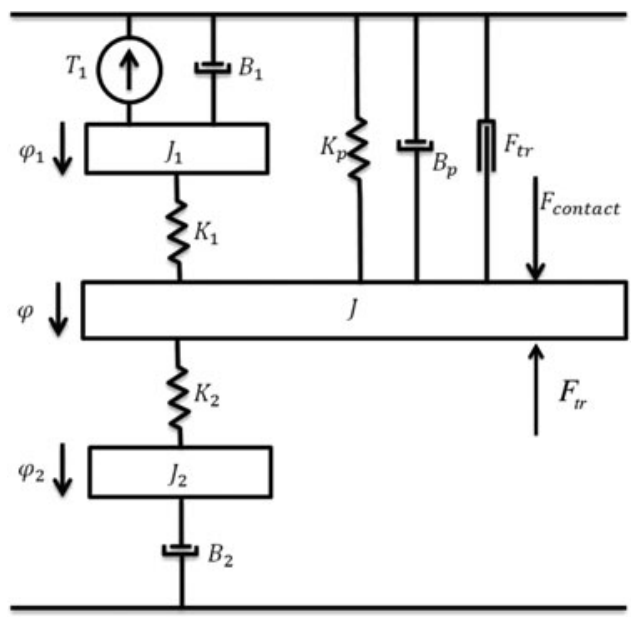

Fig. 5 An alternative representation of the bio-mechanical model of the human lower arm.

rather small modeling errors. The resulting contact-free model is

$$
\begin{aligned}
J_{1} \ddot{\varphi}_{1} & =-B_{1} \dot{\varphi}_{1}-K_{1} \varphi_{1}+K_{1} \varphi-T_{1} \\
J \ddot{\varphi} & =-B_{p} \dot{\varphi}-\left(K_{p}+2 K_{1}\right) \varphi+K_{1} \varphi_{1}+K_{1} \varphi_{2}+\underbrace{F_{g} R \cos \varphi}_{M_{b}(\varphi)}-F_{t r} \\
J_{1} \ddot{\varphi}_{2} & =-B_{1} \dot{\varphi}_{2}-K_{1} \varphi_{2}+K_{1} \varphi .
\end{aligned}
$$

Equation (2) can easily be extended to include the collision torques induced by the (orthogonal) impact.

$$
\begin{aligned}
J \ddot{\varphi} & =-B_{p} \dot{\varphi}-\left(K_{p}+2 K_{1}\right) \varphi+K_{1} \varphi_{1}+K_{1} \varphi_{2}+M_{b}-F_{t r}+M_{i m}, \\
M_{\text {im }} & =R F_{\text {tissue }} .
\end{aligned}
$$

The contact force $F_{\text {tissue }}$ contains the measurement results from Section 2.1.1 and depends on the deflection between colliding object (robot) and the human lower arm. In a more compact way, the 
Table I. Parameters of human lower arm model.

\begin{tabular}{lll}
\hline$T_{1}$ & 0.31 & {$[\mathrm{Nm}]$} \\
$B_{1}$ & 0.059 & {$[\mathrm{Nms} / \mathrm{rad}]$} \\
$K_{1}$ & 5.91 & {$[\mathrm{Nm} / \mathrm{rad}]$} \\
$J_{1}$ & $4.5 \cdot 10^{-4}$ & {$\left[\mathrm{Nms}^{2} / \mathrm{rad}\right]$} \\
$J$ & 0.07 & {$\left[\mathrm{Nms}^{2} / \mathrm{rad}\right]$} \\
$B_{p}$ & 0.431 & {$[\mathrm{Nms} / \mathrm{rad}]$} \\
$K_{p}$ & 1.86 & {$[\mathrm{Nm} / \mathrm{rad}]$} \\
$F_{t r}$ & 0.5 & {$[\mathrm{Nm}]$} \\
$R$ & 0.15 & {$[\mathrm{~m}]$} \\
\hline
\end{tabular}

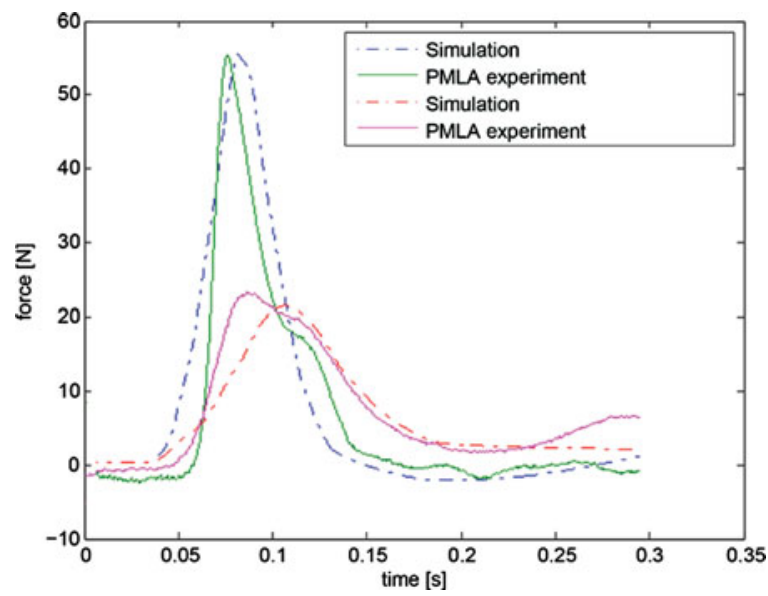

Fig. 6 Force recordings from robot-human impact simulations and experiments at $0.2 \mathrm{~m} / \mathrm{s}$ and $0.5 \mathrm{~m} / \mathrm{s}$ collision speed, respectively.

model can be represented as follows:

$$
\begin{aligned}
& {\left[\begin{array}{c}
\ddot{\varphi} \\
\ddot{\varphi}_{1} \\
\ddot{\varphi}_{2}
\end{array}\right]=\left[\begin{array}{ccc}
-B_{p} / J & 0 & 0 \\
0 & B_{1} / J_{1} & 0 \\
0 & 0 & -B_{1} / J_{1}
\end{array}\right]\left[\begin{array}{c}
\dot{\varphi} \\
\dot{\varphi}_{1} \\
\dot{\varphi}_{2}
\end{array}\right]+} \\
& {\left[\begin{array}{ccc}
-\left(K_{p}+2 K_{1}\right) / J & K_{1} / J & K_{1} / J \\
K_{1} / J_{1} & -K_{1} / J_{1} & 0 \\
K_{1} / J_{1} & 0 & -K_{1} / J_{1}
\end{array}\right]\left[\begin{array}{c}
\varphi \\
\varphi_{1} \\
\varphi_{2}
\end{array}\right]+} \\
& {\left[\begin{array}{c}
\left(-F_{t r}+M_{i m}+M_{b}\right) / J \\
-T_{1} / J_{1} \\
0
\end{array}\right]}
\end{aligned}
$$

In principle, the bio-mechanical arm model is driven by the collision force $F_{t i s s u e}$. To determine the concrete parameter values of the mathematical lower arm model, the simulation response of our model was compared to the dynamic impact response of a robot end-effector and a human volunteer, see Fig. 6. The simulated contact force in Fig. 6 is obtained from a virtual wall (the robot) moving at a certain impact speed, the human position $x_{\text {human }}$, and the contact characteristics from Fig. 3. The robot is assumed to maximally decelerate at the moment of the collision incident. Using some basic optimization routines $B_{1}, K_{1}, B_{p}, K_{p}$, and $F_{t r}$ could be found, see Table I. $R$ denotes the distance from the elbow rotation center to the impact location and could be measured directly in the experiments. All missing parameters were taken from ref. [25].

In order to match the controller to the technical PMLA requirements, a small modification was made to Eq. (2): the term $J \ddot{\varphi}$ was replaced directly by the motor torque $T_{m o t o r}$, as the PMLA physically 


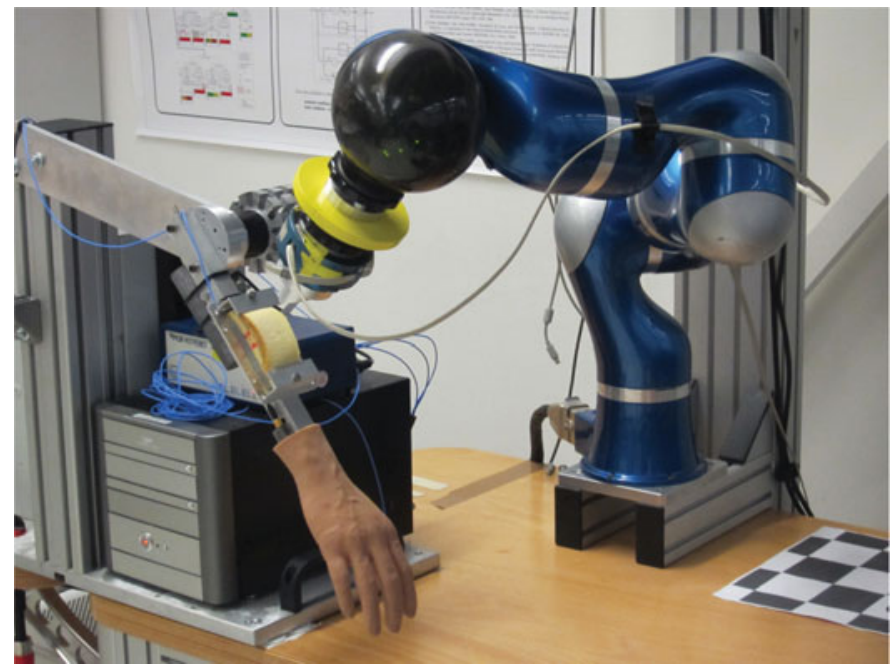

Fig. 7 Collision setup for LWR-III/PMLA experiments.

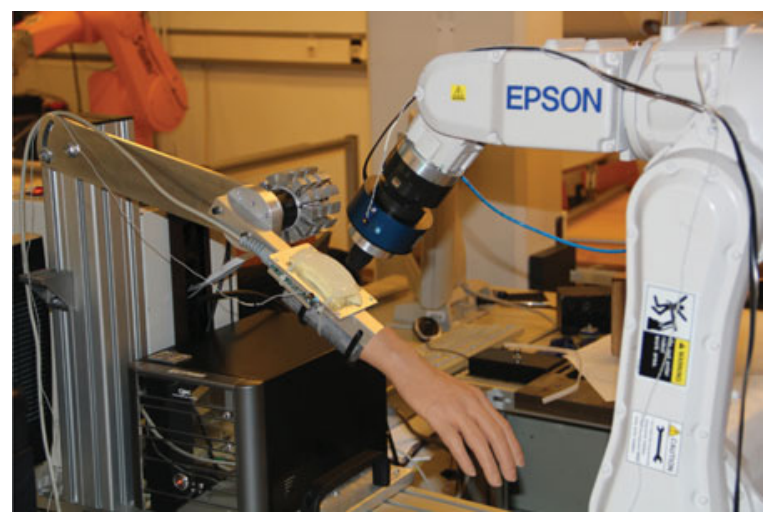

Fig. 8 Collision setup for industrial robot/PMLA experiments.

resembles a rigid body with inertia $J$. In the final controller design, Eq. (3) is thus replaced by

$$
T_{m o t o r}=-B_{p} \dot{\varphi}-\left(K_{p}+2 K_{1}\right) \varphi+K_{1} \varphi_{1}+K_{1} \varphi_{2}+M_{b}-F_{t r}
$$

\subsection{Setup PMLA/human volunteer impact experiments}

Several impact experiments were carried out with the PMLA and a human volunteer in order to validate the impact responses of the PMLA and simultaneously generate experimental human impact response data. The experiments were performed with the LWR-III and a standard industrial EPSON PS3L robot, see Figs. 7, 8. In the experiments, three end-effectors (plane, line (radius $3 \mathrm{~mm}$ ), and point (radius $2 \mathrm{~mm}$ )) were used, see Fig. 9. Many robot end-effector tools found in industry are composed of aforementioned end-effector geometries, see Fig. 10. The geometries are well-established curvature primitives found in almost every possible industrial pHRI application. The considered interaction situation is where the human may accidentally intrude in the robot's workspace, and the device collides with the upper extremities of the worker. In the experiment, the robot end-effector was displaced towards the impact point positioned on the lower arm (the human lower arm or the PMLA) with a constant velocity incrementally rising after each impact by $0.1 \mathrm{~m} / \mathrm{s}$. The range of end-effector velocities was $v=0.1: 0.1: 1 \mathrm{~m} / \mathrm{s}$. The lower arm was positioned at $35^{\circ}$ with respect to the horizontal axis. A force sensor was rigidly mounted between the end-effector and the robot. Recordings were taken at rate of $1 \mathrm{kHz}$. There was no need for a high-speed force sensor, considering the soft material against which the impacts were performed. A force/torque sensor JR3 90M32A-I50 with a corner frequency of $300 \mathrm{~Hz}$ was used. A high-speed accelerometer (PCB Piezotronics 356A02) was 


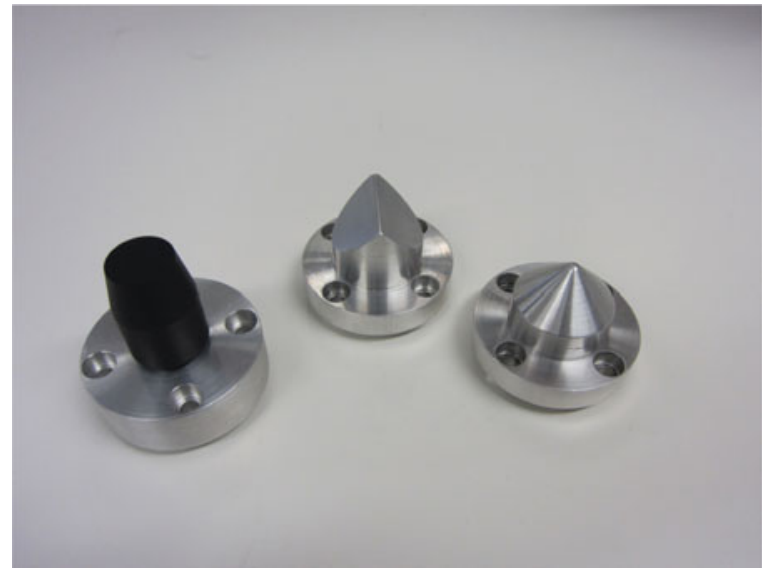

Fig. 9 Differently shaped industrially relevant robot end-effectors for plane, line, and point impact.

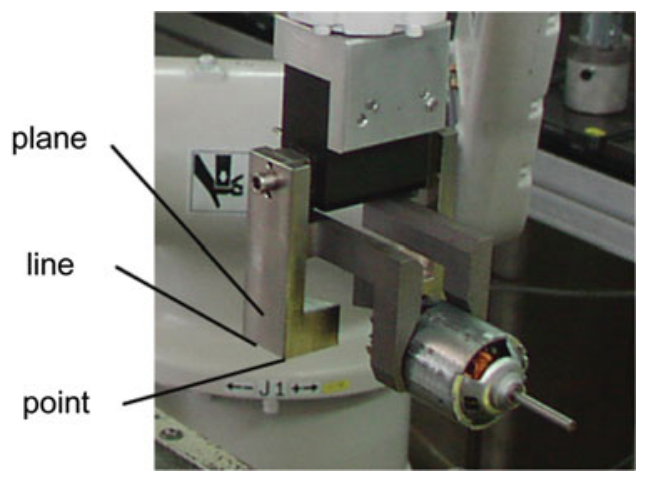

Fig. 10 Industrial robot tool composed of basic geometry shapes (plane, line, point).

attached to the PMLA-supporting aluminium profile or the volunteer's lower arm. The data from the accelerometer were logged by the XPC target computer at $1 \mathrm{kHz}$, while the JR3 force sensor data were recorded by the robot controller. Data synchronization was provided via timestamp logging. In the experiments performed with the LWR-III robot, additional weights of the robot end-effector varied between $1 \mathrm{~kg}$ and $5 \mathrm{~kg}$ by $1 \mathrm{~kg}$ increments for each end-effector experiment.

Next, we introduce the injury indicators used for estimating the occurrence of hematoma and lacerations. First, we describe the measurement procedure for assessing the IED and subsequently the one for tensile stress.

\subsection{Impact energy density}

A well-established biomechanical/forensic measure for assessing the occurrence of hematoma is the IED, which is defined as

$$
e_{A}=\frac{1}{A_{\mathrm{EE}}} \int_{\mathrm{x}_{0}}^{\mathrm{x}_{1}} F(s) \mathrm{d} s .
$$

Generally, higher IED causes injury at a higher level. ${ }^{20}$ More specifically, impacts causing higher impact energy densities than $2.52 \mathrm{~J} / \mathrm{cm}^{2}$ may cause tissue damage (e.g. hematoma). ${ }^{20,21} \mathrm{In} \mathrm{Eq.} \mathrm{(8),} F$ is the normal impact force applied to the lower arm; $x_{0}$ and $x_{1}$ denote the displacement between the robot end-effector and the lower arm bone tissue at impact start time $t_{0}$ and end time $t_{1} . A_{E E}$ is the average contact surface area between the robot end-effector and the lower arm surface. $A_{E E}$ is determined using Fujifilm Prescale pressure foil (see Section 2.5) and $F(s)$ by a JR3 force/torque sensor. The integration interval $\left[\begin{array}{ll}x_{0} & x_{1}\end{array}\right]$ was determined by calculating the distance between the robot end-effector and the lower arm. The robot end-effector movement was recorded by the robot controller, and the lower arm displacement was obtained by integration of the lower arm acceleration (PCB Piezotronics 


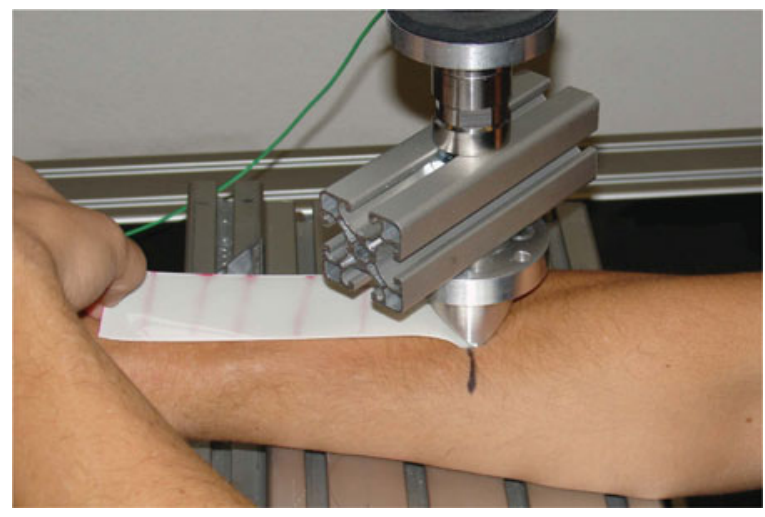

Fig. 11 Experimental setup for the impact contact area evaluation.

356A02). This approach is sufficiently accurate for the very short duration considered. Kinetic energy is transferred from the robot to the lower arm tissue only from the moment on the end-effector touches the lower arm $\left(x_{0}\right)$ until the distance between the end-effector and lower arm bone surface is smallest $\left(x_{1}\right)$. This time usually ranges between $30 \mathrm{~ms}$ and $100 \mathrm{~ms}$ for the impacts evaluated in the experiment. Thereafter, the kinetic energy of the robot is transferred to lower arm motion, since the robot endeffector starts to push it significantly. ${ }^{23}$ The energy absorbed by the tissue divided by the contact surface area thus represents the IED (8). However, the energy density can only be used to evaluate contusions expressed by bruises and crushes. ${ }^{17}$ Abrasions, lacerations, and punctures have to be investigated using different criteria. They are not likely to occur during the impacts we investigated. In many cases, sharp edges and corners of robot tools could be avoided during the end-effector design.

\subsection{Tensile stress}

Tensile stress can be considered for laceration injury assessment. Impacts applying higher tensile stress to the contact area than the ultimate tensile stress of the skin can lead to skin fracture. The ultimate tensile stress for the skin is assumed to be $1 \mathrm{MPa}^{28}$ The tensile stress applied to the soft-tissue of the lower arm (foam for the PMLA) during the impact was evaluated as follows:

$$
\begin{aligned}
\sigma_{c, \max } & =\frac{F_{\max }}{A}, \\
\sigma_{t, \max } & =\frac{\sigma_{c, \max }(1-2 v)}{3} .
\end{aligned}
$$

In Eq. (9), $F_{\max }$ represents the maximum force, and $A$ represents the average contact surface area between the robot end-effector and the lower arm. For the maximum tensile stress calculation, an assumption was made that the foam representing the soft tissue has the same Poisson ratio as the soft tissue of the human lower arm $(v=0.42){ }^{29}$

\subsection{Estimated impact contact area}

The contact area used for obtaining the tensile stress and IED was $3.46 \mathrm{~cm}^{2}$ for the plane impact. This value was derived by measuring the top surface of the cylindrically-shaped plane end-effector, see Fig. 9. The contact area for the line and point impactor was determined in a quasi-static experiment with FujiFilm pressure foil, see Fig. 11. The end-effector (line, point) was pressed against the volunteer's lower arm at various quasi-static contact forces $(20 \mathrm{~N}, 40 \mathrm{~N}, 60 \mathrm{~N}, 80 \mathrm{~N}, 100 \mathrm{~N})$. The pressure foil was positioned between the end-effector and the lower arm, see Fig. 11. Dedicated hardware and software equipment was used for subsequent pressure foil analysis. Each imprint was evaluated in terms of the highest pressure. The contact area was assumed to be constant with increasing force, see Fig. 12. The contact area was obtained from the respective regression line. The contact area was $A=1.22 \mathrm{~cm}^{2}$ for the line end-effector and $A=0.234 \mathrm{~cm}^{2}$ for the point end-effector. An error is introduced only for lower force values ranging from $0 \mathrm{~N}$ to $20 \mathrm{~N}$. This error is not significant since the impacts resulting from such low impact forces were not the focus of our investigation. 


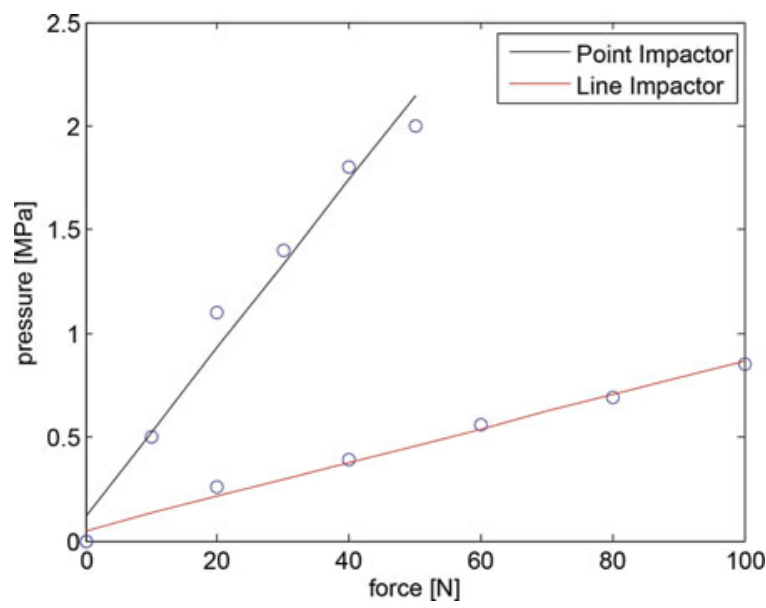

Fig. 12 Pressure values measured with FujiFilm pressure foil at various quasi-static contact forces.

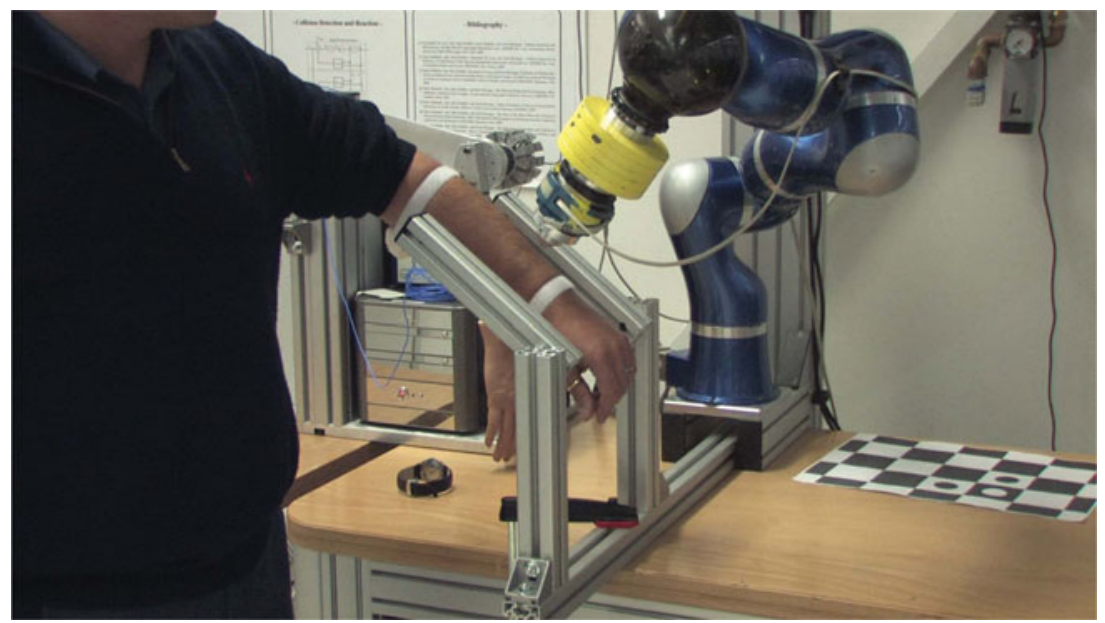

Fig. 13 Experimental setup for LWR-III/human volunteer impact experiments.

\section{Experimental Results}

In this section, we present the experimental results of our impact study, the relevant comparison to the PMLA device, and the generalization to full-scale safety curves based on a collision series with varying impact mass (LWR-III with additional masses mounted on the end-effector) and velocity with the verified PMLA.

\subsection{Human lower arm verification experiments}

The initial IED limit of $2.52 \mathrm{~J} / \mathrm{cm}^{2}$ was determined by cadaver experiments and not with testing on living tissue. ${ }^{21}$ Furthermore, the literature IED limit is valid for thin tissues with underlying bone close to the skin. One goal of this investigation was also to evaluate this limit for muscle, adipose tissue, and skin surrounding the bones of the lower arm (ulna, radius), and soft-tissue of the upper side of the hand. A short overview of resulting quantities and according impact locations is provided in Figs. 14-16. The experiments included different impact points positioned on the dorsal aspect of the lower arm ( 3 impact points), ventral aspect of the lower arm ( 2 impact points), and on the hand (4 impact points). For each impact point, one perpendicular impact was carried out with robot end-effector displaced at constant velocity of $1 \mathrm{~m} / \mathrm{s}$. The robot was equipped with a point aluminium end-effector, see Fig. 13. After each impact, the volunteer estimated the pain felt during the impact using a scale, ranging from 0 to 100 (0-20 no pain, 20-40 mild pain, 40-60 moderate pain, 60-80 horrible pain, 80-100 unbearable pain). Twelve hours after the experiment, the volunteer was asked to estimate the touch sensitivity at impact points in terms of the visual analog scale (VAS) ${ }^{30-32}$ as 


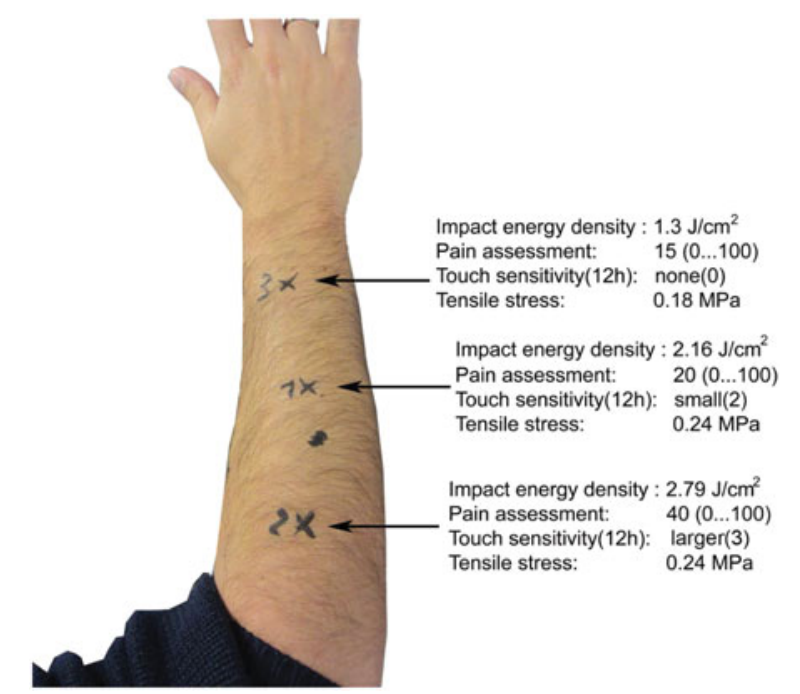

Fig. 14 Three impact locations on the dorsal aspect of the human lower arm.

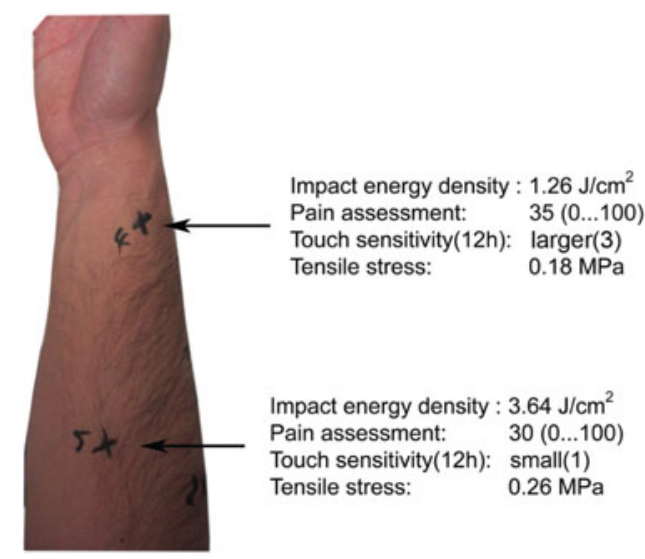

Fig. 15 Two impact locations on the ventral aspect of the human lower arm.

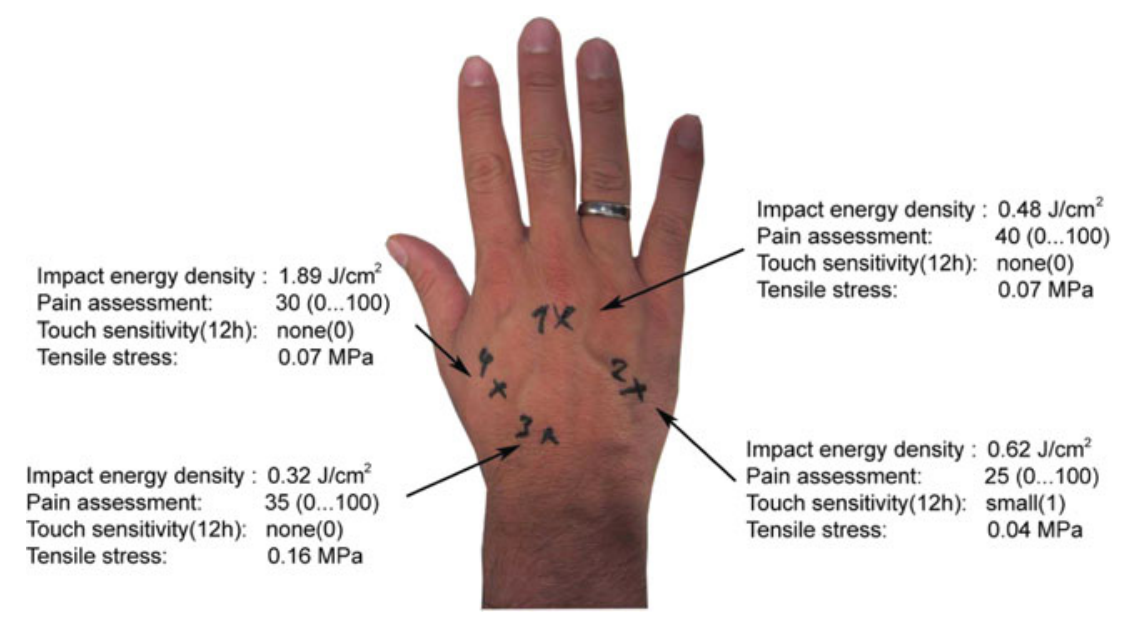

Fig. 16 Four impact locations on the human hand. 


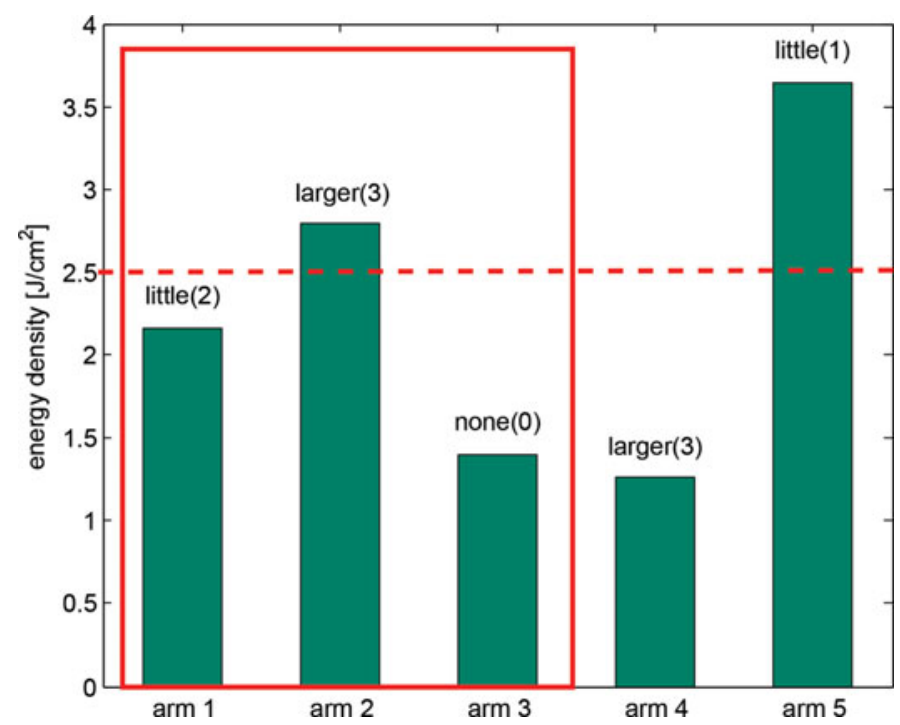

Fig. 17 Impact energy density and touch sensitivity for lower arm impacts.

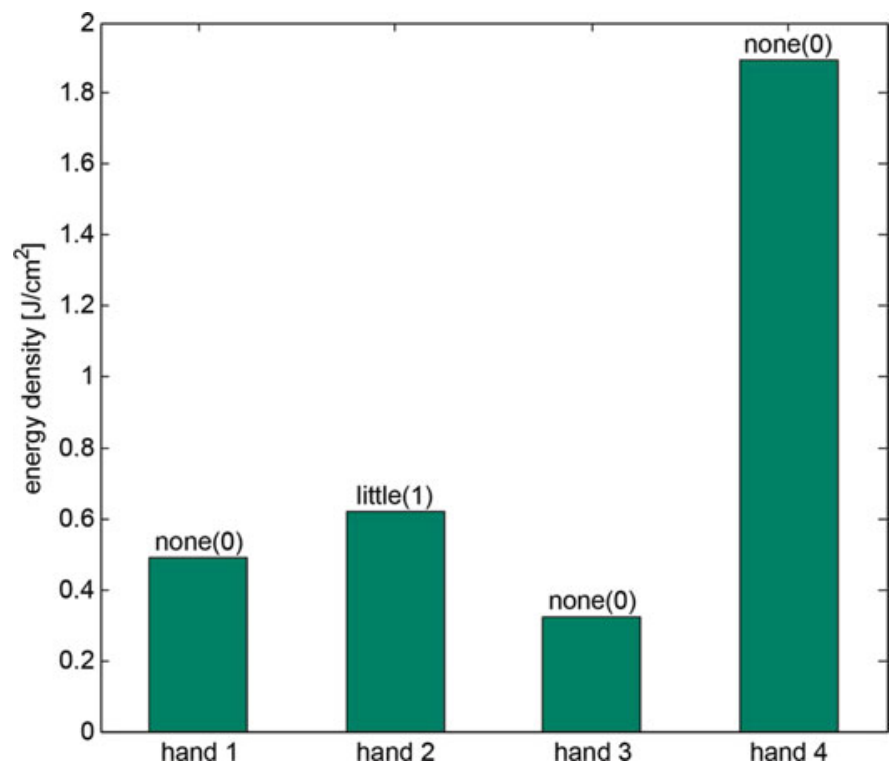

Fig. 18 Impact energy density and touch sensitivity for hand impacts.

an indicator for mild contusions. The analog scale ranging from 0 to 10 was used with descriptive values: none (0), little (1), medium (2), larger (3), not used (4-10).

Experimental results for the IED of the lower arm and hand impacts are depicted in Figs. 17 and 18. Above the bars, which represent the IED, the touch sensitivity at the impact points on the lower arm and hand that was reported by the subject after $12 \mathrm{~h}$ is denoted. Red rectangles mark the areas that are most relevant in the dorsal aspect of the lower arm. The limit of $2.52 \mathrm{~J} / \mathrm{cm}^{2}$ is marked with a dashed line. Twelve hours after the experiment, the volunteer reported little touch sensitivity at the first impact point with the IED below the dashed line. At the second impact point, the impact energy density surpassed the limit, marked by the dashed line, and the reported touch sensitivity was higher. Similarly to the first impact point, the volunteer reported little touch sensitivity at the third impact point with the IED below the dashed line. More extensive research in the area of soft-tissue injuries caused by impacts with differently shaped end-effectors is planned for generating further insights. Nevertheless, from our rather initial investigation, we can conclude that the limit of $2.52 \mathrm{~J} / \mathrm{cm}^{2}$ could be valid for mild contusions in the dorsal area of the lower arm. 

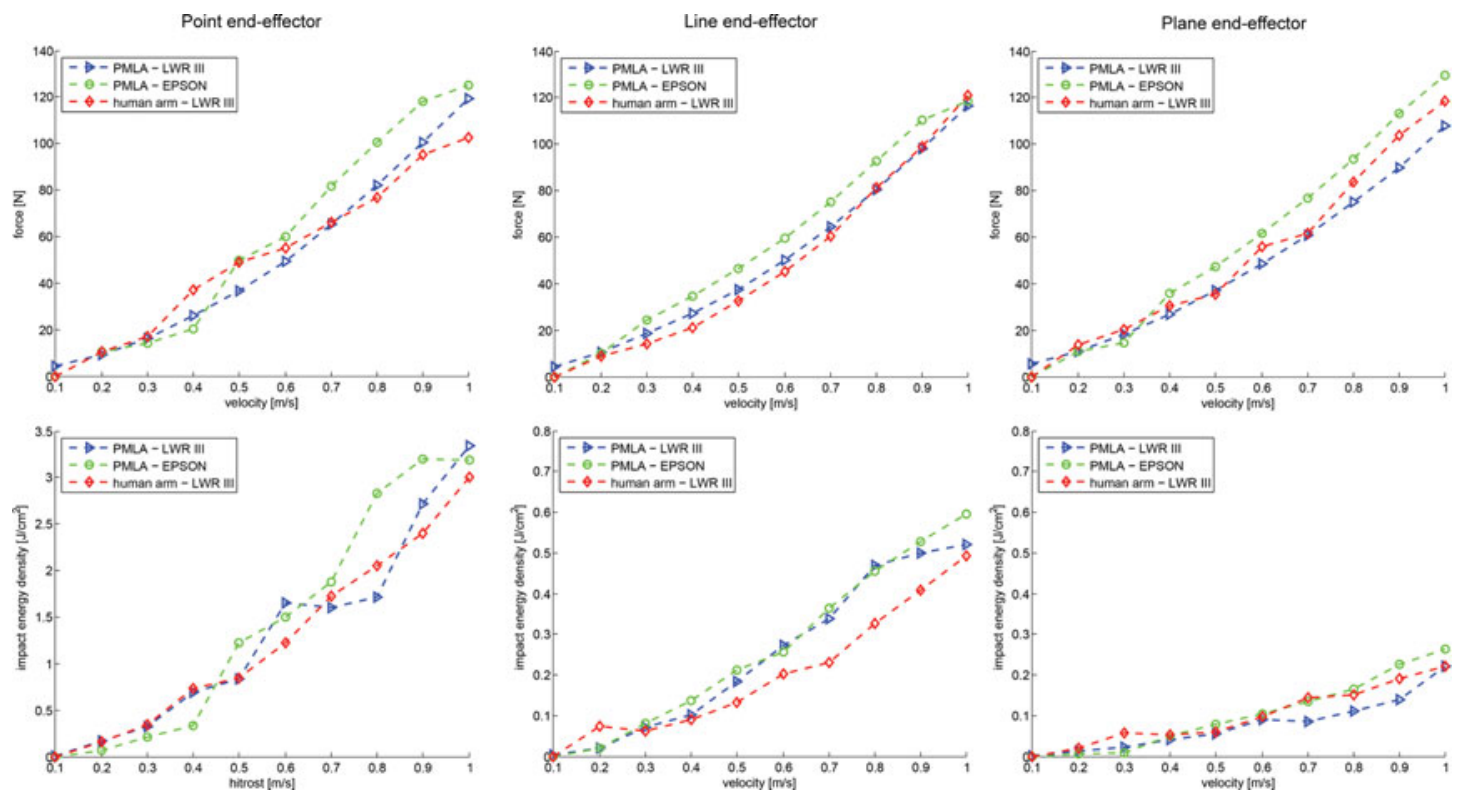

Fig. 19 Impact forces and impact energy densities with respect to the robot end-effector velocity for point (left two figures), line (two figures in the middle), and plane end-effector (two figures on the right).

\subsection{Comparison of PMLA and human volunteer}

Figure 19 depicts the experimental comparison of different impact experiments (LWR-III and PMLA, EPSON robot and the PMLA, LWR-III and human volunteer) up to a collision velocity of $1 \mathrm{~m} / \mathrm{s}$. The first row shows the impact forces and the second one impact energy densities, both as functions of end-effector velocity. Each column represents the results for one of the aforementioned end-effectors. The first column shows the results with the point end-effector, the second the ones with the line end-effector, and the third results with the plane end-effector. The impact forces for the three different experiments are similar for the different robot end-effectors and reached maximal values ranging between $100 \mathrm{~N}$ and $125 \mathrm{~N}$. In general, the impact forces are slightly higher for the impacts performed with the industrial robot and the PMLA. Rather small differences can be noticed when comparing the impact forces for the LWR-III with either the PMLA or the human volunteer. The substantial differences in impact energy densities between the different end-effectors, clearly originating from the widely varying contact characteristics, are noteworthy. Maximal values of $\approx 3.3 \mathrm{~J} / \mathrm{cm}^{2}$ can be observed for the point end-effector at $1 \mathrm{~m} / \mathrm{s}$ impact velocity. Impact energy densities evaluated with the line end-effector are significantly lower $\left(\approx 0.55 \mathrm{~J} / \mathrm{cm}^{2}\right)$. The lowest values are found for the plane impact experiments and range between $0.22 \mathrm{~J} / \mathrm{cm}^{2}$ and $0.28 \mathrm{~J} / \mathrm{cm}^{2}$.

\subsection{Safety curve}

As already outlined, a further aim of our experimental investigation is to contribute more complete experimental injury data that could be exploited in the unified treatment introduced in ref. [18]. The idea of this recent methodology is to generate biomechanically safe robot velocities by using so-called safety curves as dynamic robot velocity constraints. The theoretical basis is that given any relevant point of interest (POI) along the robot structure (including its end-effector) that constitutes a certain local surface curvature, one may calculate the POI's safe velocity such that human injury thresholds are not exceeded during a potential collision with a human body. This is done by evaluating the POI's reflected mass at a given joint configuration and obtain the respective (dynamic) velocity limit from the safety curve representation. This representation shows the injury/pain threshold curve for a certain injury (indicator) in the (reflected mass, velocity)-plane, which is inherently robot independent. Such curves can be obtained either from basic bio-mechanical testing (such as drop testing or pendulum testing of biological tissue or human subject testing), robot impact testing in which the robot's reflected impact inertia is known (as is the case in this paper), or from suitable biomechanically verified impact simulations. Here, we consider the IED limit of $2.52 \mathrm{~J} / \mathrm{cm}^{2}$ as a suitable injury indicator for hematoma and the tensile stress for lacerations. Furthermore, the maximum impact 

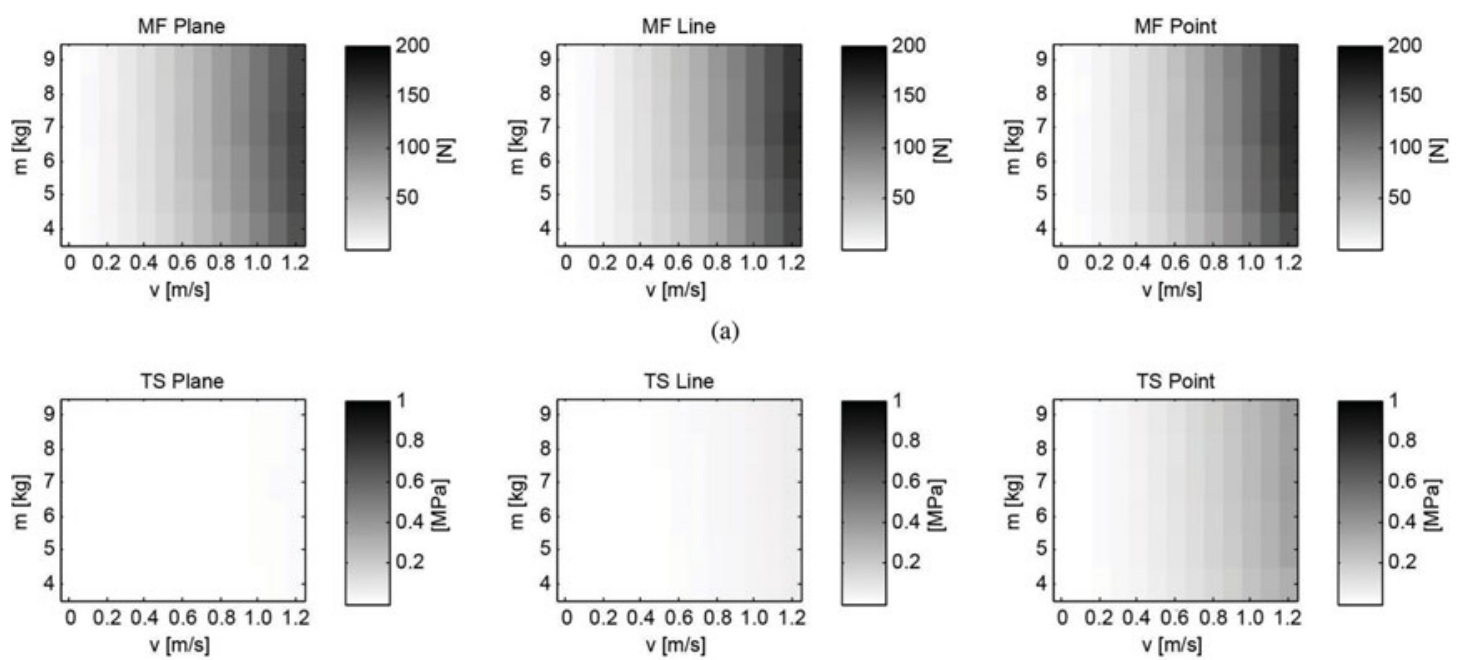

(b)
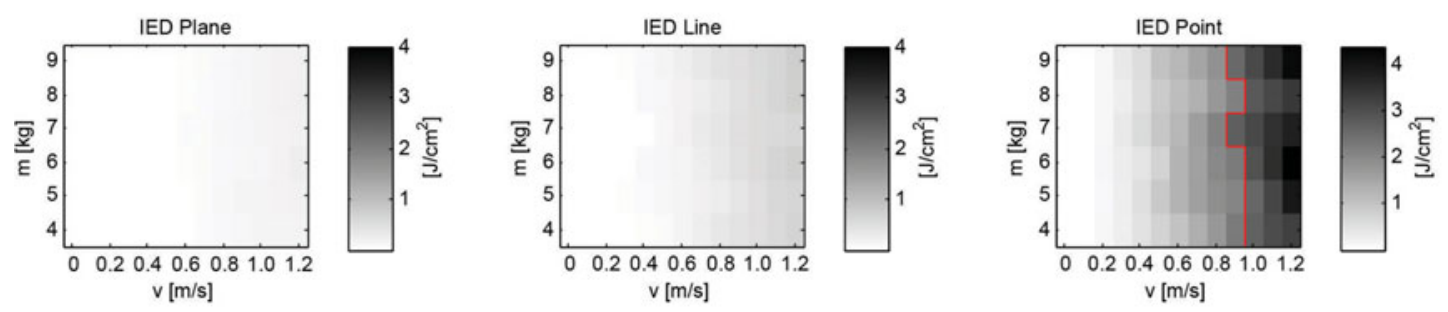

(c)

Fig. 20 Reflected mass-velocity dependency of maximum impact force (upper row), tensile stress (middle row), and impact energy density (last row). The first column depicts the results for the plane end-effector, the second for the line end-effector, and the third for the point end-effector.

force is depicted for reference and better intuition. In order to generate the threshold curves in the (reflected mass, velocity)-plane, a series of systematic impact experiments with the validated PMLA was conducted with the LWR-III. The experimental setup was the same as described in Section 2.2. The range of considered end-effector velocities is $v=0.1: 0.1: 1.2 \mathrm{~m} / \mathrm{s}$. In Fig. 20 (row one to three), the weight-velocity-force/tensile stress/IED scale relations are depicted for 216 impact tests. Each end-effector (plane, line, and point) occupies one column, showing the maximum impact force, tensile stress and IED. The maximum force/tensile stress/impact energy density is equivalent to the grayscale rectangular fields. Black rectangular fields represent the highest measured value and white the value zero. Plane end-effector results are depicted in the upper row of Fig. 20. The left grayscale represents the impact force with respect to impact velocity and the robot's reflected inertia along impact direction. Obviously, the maximum impact force rises with increasing end-effector velocity. In contrast, increasing robot reflected inertia causes only slight increases of the measured impact force. This effect was already documented in ref. [15] and is related to the inertial saturation effect. In our investigation, the impacts with plane end-effector generated maximum tensile stress of $0.02 \mathrm{MPa}$, which is substantially below the threshold of $1 \mathrm{MPa}$. This indicates that lacerations, which are indicated by tensile stress, are not likely to occur at such an impact. Impact energy densities, which are accepted bio-mechanical injury indicators for contusions, were below the threshold of $2.52 \mathrm{~J} / \mathrm{cm}^{2}$ for the plane end-effector. Similarly to the maximum impact force and maximum tensile stress, the robot's reflected inertia has only minor influence on the IED. Maximum force results for the line end-effector are similar to the plane end-effector with slightly higher maximum impact forces. The impact forces depend, however, more on the end-effector velocity than on the reflected robot inertia. Tensile stress evaluated in the impact experiments with the line end-effector is again substantially below the injury threshold of $1 \mathrm{MPa}$. All observed impact energy densities are below $2.52 \mathrm{~J} / \mathrm{cm}^{2}$ for the evaluated end-effector velocities and robot reflected inertias. Maximum impact forces measured with the point end-effector are depicted in the first row, third column of Fig. 20. Impact experiment results with the point end-effector show similar results as for the line end-effector 


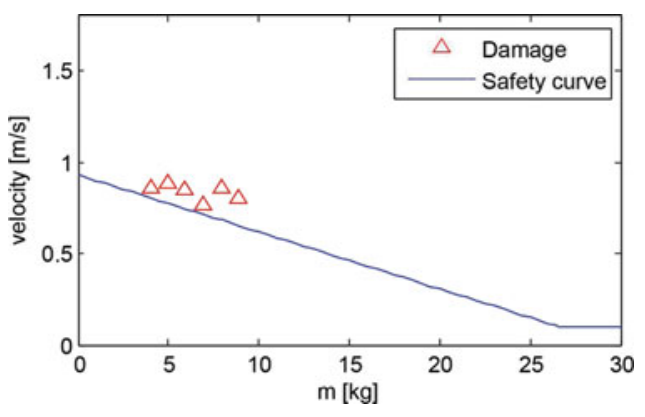

Fig. 21 Conservative safety curve for the point robot end-effector.

in terms of maximum force. The maximum values of the tensile stress with the point end-effector are substantially higher than with the plane and line end-effectors. However, the maximum values $(0.35 \mathrm{MPa})$ are still quite below the injury threshold of $1 \mathrm{MPa}$. The third row of the third column in Fig. 20 represents the IED for the point end-effector with respect to impact velocity and reflected inertia. The red line marks the limit of $2.52 \mathrm{~J} / \mathrm{cm}^{2}$. Impacts causing higher impact energy densities may in turn result in tissue damage (e.g. in terms of mild contusions) without lacerations since the tensile stress is below $1 \mathrm{MPa}$.

Transitive values marked by the red line in the third row of the third column in Fig. 20 were used to generate a safety curve for the aforementioned safe robot velocity shaping, see Fig. 21. This reduced representation is obtained by simple conservative regression fitting and limiting the minimum/maximum velocity to reasonable values, if necessary. In this schema, triangles represent the impact velocities at which tissue damage could occur in principle, and the line represents safe end-effector velocities for a given primitive (in this case the point end-effector). The safe velocity limit was chosen below the points marking potential soft tissue injury thresholds. On one side, the velocity limit establishes a maximum velocity of $0.95 \mathrm{~m} / \mathrm{s}$ for robot reflected inertias close to zero and on the other side it extends to a predefined value of $0.1 \mathrm{~m} / \mathrm{s}$ for high values of the reflected inertia in the displacement direction. Robots intended for safe interaction with humans are usually lightweight with reflected inertias in the range of $1 \mathrm{~kg}-15 \mathrm{~kg}$. However, near singularities the robot reflected inertia rises rapidly. It seems reasonable to set a predefined velocity for high reflected inertias in order to prevent the robot from stopping near singularities, similar to ref. [18]. Alternatively, momentum-based limits might be selected instead.

\section{Conclusion}

In this paper, an actively controlled mechanical lower arm was developed for comparative analysis of human-robot impact studies. Several impact experiments were carried out with the PMLA and a human volunteer in order to validate the impact responses of the device. The elbow joint was controlled with a model based controller that mimics the impedance behavior of a human elbow. Overall, the experimental results clearly show that the PMLA is a reasonably good injury estimation system for unconstrained lower arm collisions. In particular, hematoma and lacerations can be analyzed with the device. Two very different robots were used to validate this statement: the LWR-III collaborative system and an EPSON PS3L industrial robot.

Some further conclusions can also be drawn from our investigation: if a robot that is equipped with a point end-effector collides with the human lower arm at $\geq 0.8 \mathrm{~m} / \mathrm{s}$, our results indicate that mild contusions may occur. The line and plane end-effector are not likely to cause any tissue damage when colliding against an unconstrained lower arm at velocities $\leq 1 \mathrm{~m} / \mathrm{s}$.

Finally, the PMLA was also used to determine new safety curves for safe robot velocity profile shaping. These can be used to determine biomechanically safe robot velocities by taking into account the robot's reflected inertia, velocity, and impact geometry. Our novel data can then be seamlessly embedded into the SMU framework from ref. [18].

Note that the human impact responses shown in this paper belong to a single volunteer, which were, however, carried out for the first time. By definition, a single subject has limiting generalization properties. However, we believe this kind of study is crucial to show that, in principle, it is possible 
to replace human subject testing with adequately controlled mimicking devices. The cross-subject validity of our data still has to be proven with more human subject experiments.

\section{Acknowledgments}

This work was supported in part by the European Union. "Operation part financed by the European Union, European Social Fund.” This work has been partially funded by the European Commission's Sixth Framework Programme as part of the projects SAPHARI under grant no. 287513.

\section{References}

1. A. Albu-Schäffer, S. Haddadin, C. Ott, A. Stemmer, T. Wimböck and G. Hirzinger, "The DLR lightweight robot - lightweight design and soft robotics control concepts for robots in human environments," Indust. Robot J. 34(5), 376-385 (2007).

2. W. Townsend and J. Salisbury, "Mechanical Design for Whole-Arm Manipulation," In: Robots and Biological Systems: Towards a New Bionics? (P. Dario, G. Sandini and P. Aebischer, eds.) (Springer, Berlin Heidelberg, 1993) pp. 153-164.

3. C. Fitzgerald, "Developing Baxter," IEEE International Conference on Technologies for Practical Robot Applications (TePRA), Woburn, MA, USA (Apr. 2013) pp. 1-6.

4. G. Hirzinger, J. Butterfaß, M. Fischer, M. Grebenstein, M. Hahnle, H. Liu, I. Schäfer and N. Sporer, "A Mechatronics Approach to the Design of Light-Weight Arms and Multi-Fingered Hands," IEEE Conference on Robotics and Automation (ICRA), San Francisco, CA, USA (2000) pp. 46-54.

5. A. D. Luca, A. Albu-Schäffer, S. Haddadin and G. Hirzinger, "Collision Detection and Safe Reaction with the DLR-III Lightweight Manipulator Arm," IEEE/RSJ International Conference on Intelligent Robots and Systems (IROS2006), Beijing, China (2006) pp. 1623-1630.

6. S. Haddadin, A. Albu-Schäffer, A. D. Luca and G. Hirzinger, "Evaluation of Collision Detection and Reaction for a Human-Friendly Robot on Biological Tissues," International Workshop on Technical Challenges and Dependable Robots in Human Environments (IARP 2008), Pasadena, USA (2008).

7. M. Grebenstein et al., "The DLR Hand Arm System," IEEE International Conference on Robotics and Automation (ICRA). IEEE, Shanghai, China (2011) pp. 3175-3182.

8. N. G. Tsagarakis, S. Morfey, G. Medrano Cerda, L. Zhibin and D. G. Caldwell, "Compliant Humanoid Coman: Optimal Joint Stiffness Tuning for Modal Frequency Control," IEEE International Conference on Robotics and Automation (ICRA). IEEE, Karlsruhe, Germany (2013) pp. 673-678.

9. S. Haddadin, A. Albu-Schäffer and G. Hirzinger, "Safety Evaluation of Physical Human-Robot Interaction via Crash-Testing," Robotics: Science and Systems Conference (RSS2007), Atlanta, USA (2007) pp. 217224.

10. N. Najmaei, "Applications of artificial intelligence in safe human-robot interactions," IEEE Trans. Syst. Man Cybern. B 41(2), 448-459 (2011).

11. K. Ikuta, H. Ishii and M. Nokata, "Safety evaluation method of design and control for human-care robots," Int. J. of Robot. Res. 22(5), 281-298 (2003).

12. J. Heinzmann and A. Zelinsky, "Quantitative safety guarantees for physical human-robot interaction," Int. J. Robot. Res. 22(7-8), 479-504 (2003).

13. H. O. Lim and K. Tanie, "Human safety mechanisms of human-friendly robots: Passive viscoelastic trunk and passively movable base," Int. J. Robot. Res. 19(4), 307-335 (2000).

14. Y. Yamada, Y. Hirasawa, S. Huand, Y. Umetani and K. Suita, "Human-robot contact in the safeguarding space," IEEE/ASME Trans. Mechatronics 2(4), 230-236 (1997).

15. S. Haddadin, A. Albu-Schäffer and G. Hirzinger, "Requirements for safe robots: Measurements, analysis and new insights," Int. J. of Robot. Res. 28(11-12), 1507-1527 (2009).

16. S. Haddadin, A. Albu-Schäffer and G. Hirzinger, "Safety analysis for a human-friendly manipulator," Int. J. Soc. Robot. 2(3), 235-252 (2010).

17. S. Haddadin, A. Albu-Schäffer, F. Haddadin, J. Roßmann and G. Hirzinger, "Study on soft-tissue injury in robotics," IEEE Robot. Autom. Mag. 18(4), 20-34 (2011).

18. S. Haddadin, S. Haddadin, A. Khoury, T. Rokahr, S. Parusel, R. Burgkart, A. Bicchi and A. Albu-Schäffer, "On making robots understand safety: Embedding injury knowledge into control," Int. J. of Robot. Res. 31(13), 1578-1602 (2012).

19. B. Povse, D. Koritnik, R. Kamnik, T. Bajd and M. Munih, "Industrial robot and human operator collision," IEEE International Conference on Systems Man and Cybernetics (SMC), Istanbul, Turkey (2010) pp. $2663-2668$.

20. S. Haddadin, A. Albu-Schäffer and G. Hirzinger, "Safe physical human-robot interaction: Measurements, analysis \& new insights," International Symposium on Robotics Research (ISRR2007), Hiroshima, Japan (2007) pp. 261-282.

21. R. Edlich, G. Rodenheaver, R. Morgan, D. Berman and J. Thacker, "Principles of emergency wound management," Ann. Emergency Med. 17(12), 1284-1302 (1988).

22. S. Haddadin, A. Albu-Schäffer and G. Hirzinger, "The role of the robot mass and velocity in physical human-robot interaction - part 1: Unconstrained blunt impacts," IEEE International Conference on Robotics and Automation (ICRA2008), Pasadena, USA (2008) pp. 1331-1338. 
23. B. Povse, D. Koritnik, R. Kamnik, T. Bajd and M. Munih, "Emulation system for assessment of humanrobot collision," Meccanica 46(6), 1363-1371 (2011).

24. P. de Leva, "Adjustments to Zatsiorsky-Seluyanov's segment inertia parameters," J. Biomech. 29(9), 1223-1230 (1996).

25. A. Trnkoczy, T. Bajd and M. Malezic, "A dynamic model of the ankle joint under functional electrical stimulation in free movement and isometric conditions," J. Biomech. 9, 509-519 (1979).

26. A. V. Hill, "The heat of shortening and the dynamic constants of muscle," Proc. R. Soc. Lond. Ser. B Biol. Sci. 126(843), 136-195 (1938).

27. S. Dorgan, "Mathematical modelling and control of human skeletal dynamics," Eng. Sci. Educ. J. 8(4), 185-192 (1999).

28. H. Yamada, Strength of Biological Materials Williams \& Wilkins in Baltimore, USA (The Williams and Wilkins Company, Baltimore, USA, 1970).

29. U. Hartmann, "Ein Mechanisches Finite-Elemente Modell des Menschlichen Kopfes," Ph.D. Thesis (University of Leipzig, Leipzig, Germany, 1999).

30. H. M. McCormack, J. David, L. Horne and S. Sheater, "Clinical applications of visual analogue scales: A critical review," Psychological Med. 18, 1007-1019, 11 (1988).

31. E. Huskisson, "Measurement of pain," Lancet 2, 1127-31 (1974).

32. W. W. Downie, P. A. Leatham, V. M. Rhind, V. Wright, J. A. Branco and J. A. Anderson, "Studies with pain rating scales," Ann. Rheum Dis. 37, 378-81 (1978). 